\title{
Computed tomographic findings of cerebral fat embolism following multiple bone fractures
}

Huong Ling $\underline{L a W}^{1}$, MD, FRCR, Siong Lung Wong ${ }^{2}$, MD, MMed, Suzet $\underline{\operatorname{Tan}}^{1}$, MBBS, MMed

ABSTRACT Fat embolism to the lungs and brain is an uncommon complication following fractures. Few reports with descriptions of computed tomographic (CT) findings of emboli to the brain or cerebral fat embolism are available. We report a case of cerebral fat embolism following multiple skeletal fractures and present its CT findings here.

Keywords: cerebral fat embolism, fat embolism syndrome

Singapore Med J 2013; 54(2): e28-e30

\section{INTRODUCTION}

Cerebral fat embolism (CFE) is an uncommon complication following fractures and is part of a wider spectrum of systemic fat embolisation. Symptoms vary from confusion to deep coma, seizures and focal neurological deficit, developing 2-3 days after initial trauma in $45 \%-85 \%$ of patients. ${ }^{(1)}$ CFE can lead to serious consequences, including disability and death.

\section{CASE REPORT}

A 21-year-old man sustained closed bilateral proximal third femoral shaft fractures, an open right olecranon comminuted fracture, a right second toe proximal phalanx fracture and a right fifth metacarpal fracture following a road traffic accident. Splinting and dorsal backslab were applied to the femoral fractures and the olecranon fracture, respectively. The patient was haemodynamically stable and there was no evidence of head injury. However, he developed sudden deterioration of consciousness and became restless and confused the next day. During this period of time, he developed a few episodes of generalised tonic-clonic seizure and subsequently became unarousable. His pupils were equal in size and reactive to light bilaterally, with no localising neurological sign. He was subsequently intubated due to his poor neurological condition. Computed tomography (CT) of the brain performed 17 hours post trauma revealed no abnormality (Fig. 1).

Physical examination revealed scattered petechiae of the upper chest and lower conjunctival folds. Blood cell counts showed a haemoglobin level of $6.5 \mathrm{~g} / \mathrm{dL}$ and a platelet count of $62,000 / \mathrm{mm}^{3}$. Blood coagulation profile was deranged, with prolongation of prothrombin time and activated partial thromboplastin time. Serum electrolytes were within the normal range. Chest radiograph showed evidence of pulmonary oedema 72 hours post trauma (Fig. 2). A second CT of the brain was done seven days post trauma for persistent impaired mental status demonstrated multiple areas of low

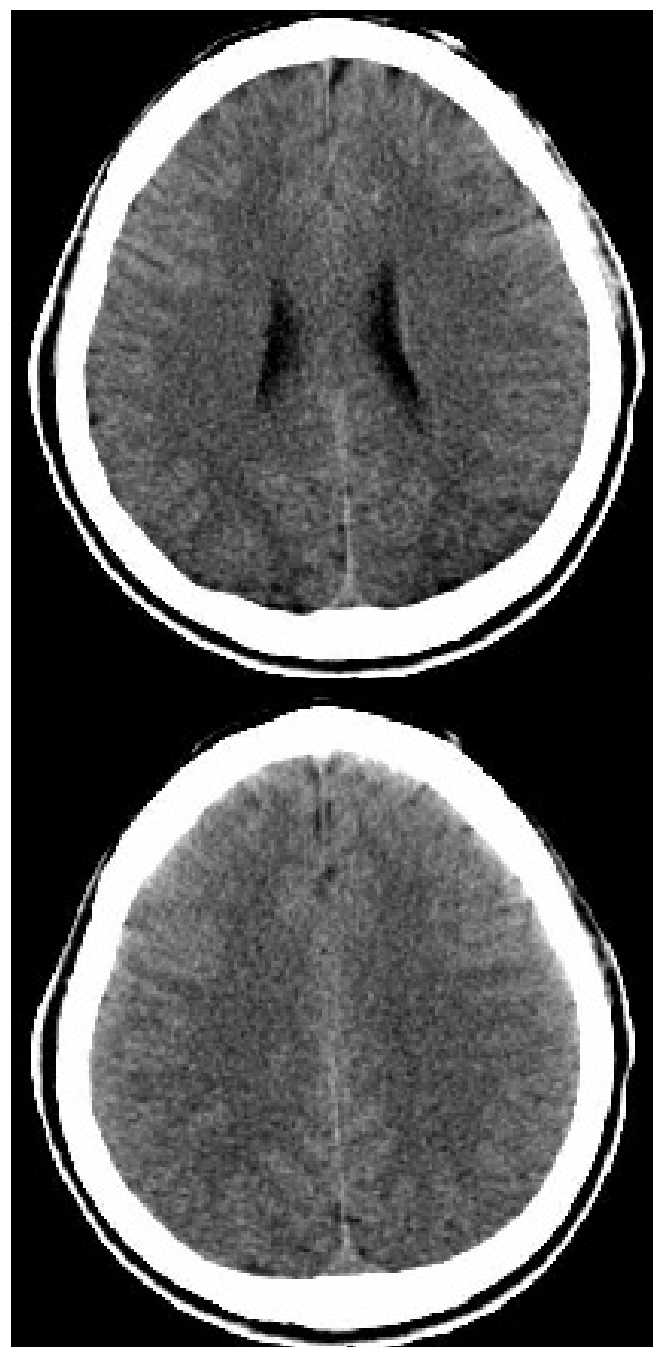

Fig. 1 Nonenhanced axial CT images of the brain obtained 17 hours after trauma show no abnormality.

attenuation within the white matter of the frontoparietal regions bilaterally (Fig. 3). He recovered gradually and was discharged from hospital one month later without any significant neurological defect.

${ }^{1}$ Department of Radiology, Sarawak General Hospital, ${ }^{2}$ Radiology Unit, University of Malaysia Sarawak (UNIMAS), Sarawak, Malaysia 

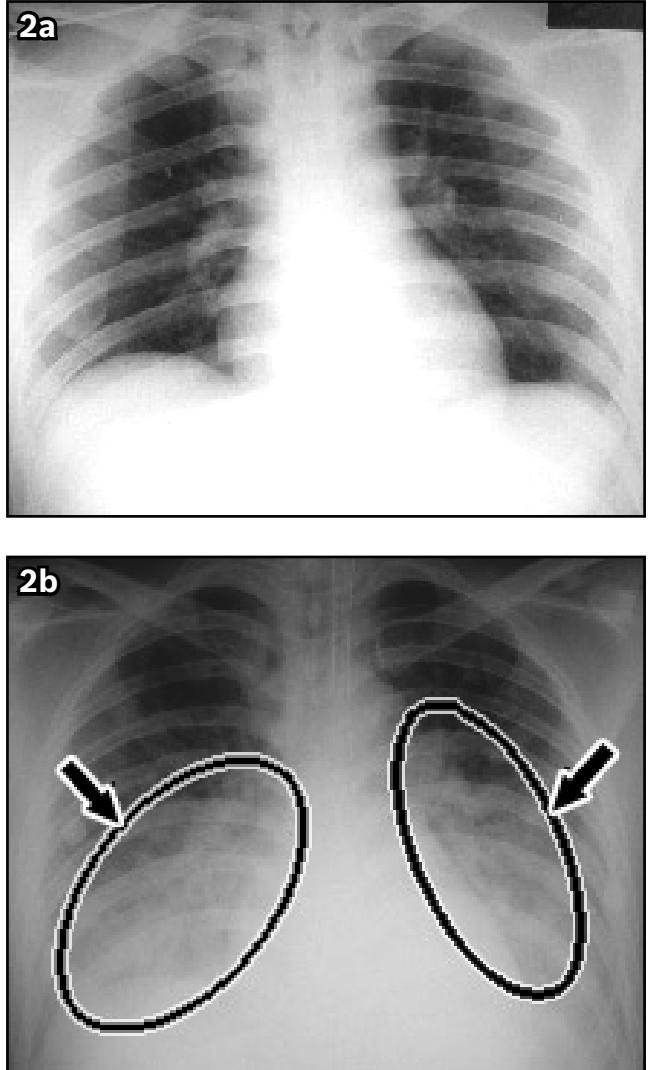

Fig. 2 (a) Chest radiograph on admission shows no abnormality. (b) Chest radiograph obtained 72 hours later shows confluent airspace opacities consistent with pulmonary oedema (circles and arrows).

\section{DISCUSSION}

Fat globules are present in pulmonary and systemic circulation in more than $90 \%$ of bone fractures. ${ }^{(2)}$ However, clinically apparent fat embolism, termed as fat embolism syndrome, is uncommon. $90 \%$ of cases are associated with bone fractures. ${ }^{(3)}$ The incidence of fat embolism syndrome ranges from $0.5 \%$ to $19 \%$ in patients with long bone fractures. The risk is higher with multiple bone fractures, lower extremities fractures or associated pelvic fractures. ${ }^{(2,4)}$ Mortality was reported to be as high as $87 \%$. $^{(4)}$

Fat embolism is a clinical diagnosis most commonly made based on Gurd's criteria. The classical triad of respiratory insufficiency, cerebral dysfunction and petechial rash is not consistently seen in all patients. Other minor criteria include fever, tachycardia, retinal changes, jaundice and renal impairment. Laboratory findings that can aid in diagnosis include anaemia, thrombocytopenia, high erythrocyte sedimentation rate and fat macroglobulinaemia. ${ }^{(3,5)}$ Clinical manifestations usually occur 12 to 24 hours after a fracture and within 72 hours of injury. ${ }^{(2,3)}$ Fat emboli that escape pulmonary vasculature and enter the systemic circulation can affect different organ systems, including the brain and lungs. Multiple theories have been postulated for the development of CFE. It has been suggested that fat globules are either liberated from the marrow of the fractured bone or form as chylomicrons coalesce in plasma. ${ }^{(3,5)}$ Subsequently, the passage of micro-fat emboli through pulmonary circulation to

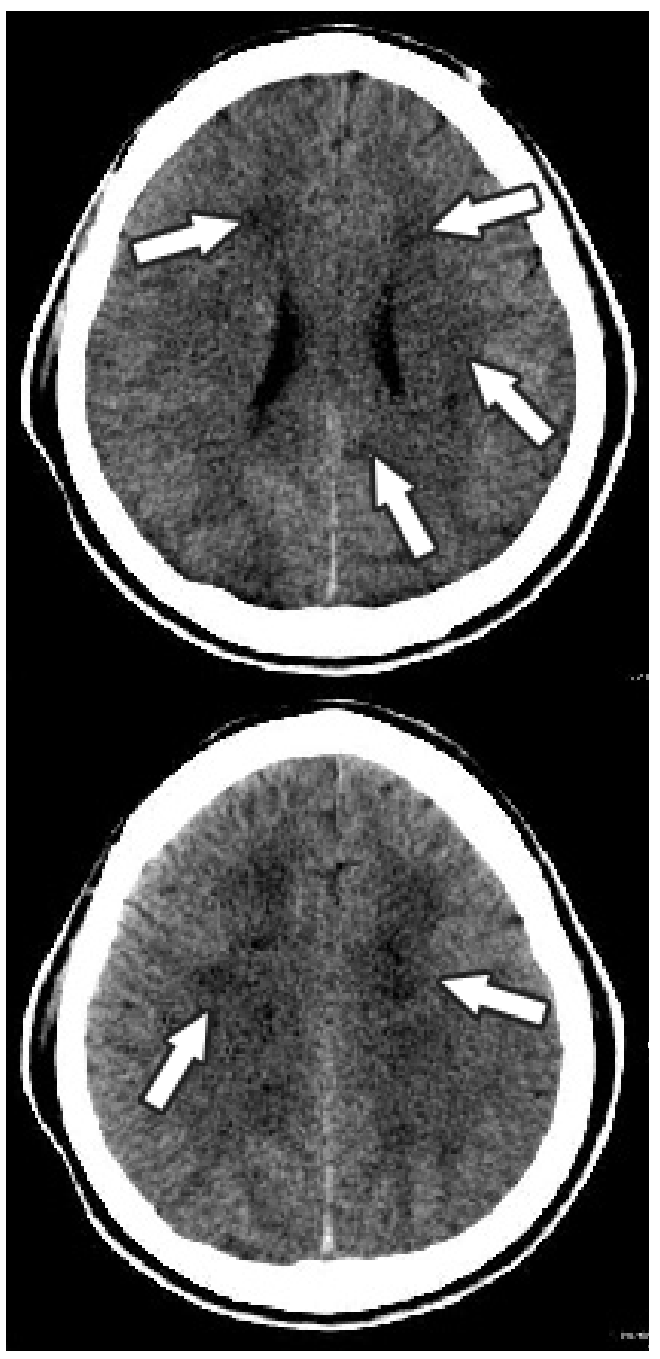

Fig. 3 Second nonenhanced axial CT images of the brain at comparative level, as that in Fig. 1 show multiple areas of low attenuation within the white matter of the frontoparietal regions bilaterally (arrows).

the cerebral capillary network causes blockage and activation of chemical mediators. ${ }^{(4)}$

Neurological manifestations are common and occur in up to $86 \%$ of patients with fat embolism syndrome. ${ }^{(6)}$ Patients usually present with variable and nonspecific neurological symptoms such as altered mental status, headache, seizure and focal neurological deficit. Clinical differentiation from other causes of neurological dysfunction is difficult. Imaging can help to rule out other pathologies, but must be interpreted in the right context. ${ }^{(6)}$ In the absence of any head injury, CFE should be suspected in trauma patients with subsequent neurological deterioration, and appropriate neuroimaging studies should be ordered.

Our patient developed neurological symptoms, in addition to pulmonary involvement and petechial rash with characteristic distribution over the upper chest and subconjunctival region, one day after sustaining multiple fractures. CFE was determined to be the most likely cause. The changes in subsequent CT images of the brain obtained seven days later confirmed the diagnosis. From our review of the literature, CT of the brain at presentation is invariably normal. Positive findings are only seen at subsequent CT images obtained from the second day onward. Reported CT 
findings in CFE are bilateral multiple low attenuation areas, diffuse cerebral oedema, subdural effusions and focal gyral enhancement. ${ }^{(6-8)}$

Magnetic resonance (MR) imaging of the brain is the most sensitive modality in the demonstration of changes in CFE and should be the first choice of imaging in suspected cases of CFE. However, CT instead of MR imaging was done in our patient, as he was seriously ill, ventilated and in need of intensive care. Characteristic MR imaging features of CFE are widely reported in the literature. In the hyperacute phase, diffusion-weighted imaging can demonstrate the starfield pattern of multiple bright spots scattered on a dark background, which is pathognomonic for CFE. (1) Subsequent MR imaging findings include multiple small, scattered T2 hyperintensities involving grey (basal ganglia and thalami) and white (subcortical and centrum semiovale) matter, which may demonstrate contrast enhancement. ${ }^{(9,10)}$ Some of these lesions may be haemorrhagic and seen as T1 hyperintensities. ${ }^{(11)}$

Patients with pulmonary fat embolism develop hypoxia with varying degrees of respiratory compromise, which may be transient or progress to adult respiratory distress syndrome. Radiographic findings of the lungs may range from normal to demonstrating patchy opacities, perihilar linear opacities (interstitial oedema), or diffuse airspace opacities (frank pulmonary oedema). ${ }^{(12)}$ In mild cases of pulmonary fat embolism where chest radiographs are normal, high resolution $\mathrm{CT}$ typically shows ground-glass opacities, thickened interlobular septa and 1-2 mm nodular opacities. ${ }^{(13)}$ The management of these patients is supportive - in an intensive care setting with ventilatory support, corticosteroids and early fracture fixation. Most patients with CFE show complete neurological recovery despite initial cerebral dysfunction and prolonged coma. ${ }^{(6,8,14)}$ This is also true in our patient, who was discharged from the hospital one month later without any neurological sequelae.

In conclusion, CFE should be considered in patients with multiple long bones fractures who subsequently develop neurological dysfunction in the absence of any head injury. In such patients, appropriate neuroimaging should be performed in order to exclude other pathologies and establish the diagnosis. MR imaging is the imaging of choice for early and accurate diagnosis of CFE. However, in cases of seriously ill and unstable patients, or where MR imaging is not available, CT is an alternative that is capable of demonstrating the intracerebral changes brought about by CFE.

\section{REFERENCES}

1. Parizel PM, Demey HE, Veeckmans G. et al. Early diagnosis of cerebral fat embolism syndrome by diffusion-weighted MRI (starfield pattern). Stroke 2001; 32:2942-4.

2. Bulger EM, Smith DG, Maier RV, Jurkovich GJ. Fat embolism syndrome. A 10-year review. Arch Surg 1997; 132:435-9.

3. Fabian TC. Unravelling fat embolism syndrome. N Engl J Med 1993; 329:961-3.

4. Lee YS, Park SH, Hamm IS. Cerebral Fat Embolism with Multiple Rib and Thoracic Spinal Fractures. J Korean Neurosurg Soc 2004; 36:408-11.

5. Gurd AR, Wilson RI. The fat embolism syndrome. J Bone Joint Surg BR 1974; 56B:408-16.

6. Jacobson DM, Terrence CF, Reinmuth OM. The neurologic manifestations of fat embolism. Neurology 1986; 36:847-51.

7. Sakamoto T, Sawada Y, Yukioka T, et al. Computed Tomography for Diagnosis and Assessment of Cerebral Fat Embolism. Neuroradiology 1983; 24:283-5.

8. Gregorakos L, Sakayianni K, Hroni D, et al. Prolonged coma due to cerebral fat embolism: report of two cases. J Accid Emerg Med 2000; 17:144-6.

9. Stoeger A, Daniaux M, Felber S, et al. MRI findings in cerebral fat embolism. Eur Radiol 1998; 8:1590-3.

10. Simon AD, Ulmer JL, Strottmann JM. Contrast-enhanced MR imaging of cerebral fat embolism: case report and review of the literature. AJNR Am J Neuroradiol 2003; 24:97-101.

11. Erdem E, Namer IJ, Saribas O, et al. Cerebral fat embolism studies with MRI and SPECT. Neuroradiology 1993; 35:199-201.

12. Curtis Am, Knowles GD, Putman CE, et al. The three syndromes of fat embolism: pulmonary manifestations. Yale J Biol Med 1979; 52:149-57.

13. Malagari K, Economopoulos N, Stoupis C, et al. High-resolution CT findings in mild pulmonary fat embolism. Chest 2003; 123:1196-201.

14. Aravapalli A, Fox J, Lazaridis C. Cerebral fat embolism and the "starfield" pattern: a case report. Cases J 2009; 2:212. 